EXPERIMENTAL STUDY

\title{
A mathematical comparison of techniques to predict biologically available testosterone in a cohort of 1072 men
}

\author{
Paul D Morris ${ }^{1,2}$, Chris J Malkin ${ }^{1}$, Kevin S Channer ${ }^{1}$ and T Hugh Jones ${ }^{1,2}$ \\ ${ }^{1}$ Department of Cardiology, Royal Hallamshire Hospital, Sheffield S1O 2JF and ${ }^{2}$ Academic Unit of Endocrinology, Division of Genomic Medicine, \\ University of Sheffield Medical School S1O 2RX, Sheffield, UK
}

(Correspondence should be addressed to Dr C J Malkin, M131, Cardiology, Royal Hallamshire Hospital, Glossop Road, Sheffield S1O 2JF, UK; Email: Chris.Malkin@sth.nhs.uk)

C J Malkin and P D Morris contributed equally to this work

\begin{abstract}
Objective: In the absence of widely available measures of determining free and/or bioavailable testosterone (BioT) physicians may use formulae such as the free androgen index (FAI) to estimate free testosterone. We compared the efficacy of calculated markers of androgen status in predicting serum BioT and hypogonadism.

Design: Total testosterone (TT), sex hormone binding globulin (SHBG) and BioT were determined in a large cohort of men. Comparison of calculated androgen levels was performed following endocrine assessment.

Methods: TT and SHBG were determined by ELISA, and BioT was determined by ammonium sulphate precipitation. From these data we calculated FAI and free testosterone using two other published formulae - FTnw (free testosterone as calculated by the method of Nanjeee and Wheeler) and FTv (free testosterone as calculated by the method of Vermeulen). A novel formula was derived to calculate BioT from given levels of TT and SHBG (BTcalculated). The ability of the methods (FAI, FTnw, FTv, BTcalc) to predict BioT were compared using regression analysis. The ability of these markers of androgen status to predict biochemical hypogonadism was compared using area under receiver operator curve (auROC).

Results: The equation derived from our data was the best predictor of BioT $\left(R^{2}=0.73, P<0.0001\right)$ although TT was also a good marker $\left(R^{2}=0.68, P=0.0001\right)$. In the determination of hypogonadism, of all currently available formulae none were better that the TT (auROC: TT $=0.93$, FAI $=0.72$, FTnw $=0.91, \mathrm{FTv}=0.88)$ although when TT is borderline $(7.5<\mathrm{TT}<12 \mathrm{nmol} / \mathrm{l})$ estimates of free testosterone are superior to TT alone (auROC: $\mathrm{TT}=0.63, \quad \mathrm{FAI}=0.74, \quad \mathrm{FTnw}=0.75$ and $\mathrm{FTv}=0.75$ ).

Conclusions: TT is the best marker of hypogonadism and BioT, when TT is borderline calculated indices of free testosterone or BioT are useful and may help confirm hypogonadism.
\end{abstract}

European Journal of Endocrinology $151241-249$

\section{Introduction}

The laboratory assessment of male androgen status has become an increasingly common request in the past decade. Physicians and the male population in general are now aware that endogenous male androgens decline with age and that the partial androgen deficiency of the ageing male may exist and require treatment. Many symptoms and complications of this clinical condition are potentially avoidable with appropriate androgen replacement therapy. Although demand for androgen therapy is increasing the ability to diagnose borderline androgen deficiency can be difficult in clinical practice. Symptomatic androgen deficiency in a young adult, supported by low serum testosterone is a clear indication for androgen replacement. In the partial androgen deficiency of the ageing male the indication for testosterone replacement is less clear, especially since there are safety concerns related to the prostate gland and an increase in erythropoiesis (1). There is currently no evidence that appropriate androgen replacement causes prostate neoplasia (2), but there is scant long-term data on the safety of androgen therapy in eugonadal or borderline hypogonadism and therefore most prescribing physicians are careful to confirm a diagnosis of androgen deficiency. On the other hand, failure to treat androgen deficiency may lead to continuing poor quality of life, marital difficulties and reduced work performance. In the long term there is an increased risk of osteoporosis and frailty with the associated socio-economic costs. The symptoms of androgen deficiency are nonspecific and unreliable and in the elderly male difficult 
to discern from generalised symptoms of ageing. Clinical impression and symptom questionnaires remain important, but a serum assessment of testosterone is mandatory for making the diagnosis.

A challenging clinical scenario is an elderly male with some symptoms of androgen deficiency but a borderline low level of total testosterone (TT). The biochemical estimation of serum testosterone in men is complicated. Testosterone shows a marked diurnal and circannual variation, and a large proportion of the hormone $(50-70 \%)$ circulates tightly bound to sex hormone binding globulin (SHBG) and is therefore physiologically inactive. A further $20-30 \%$ circulates bound loosely to albumin and only $1-3 \%$ of the hormone is free in the serum. The albumin-bound fraction and the free fraction are physiologically available to the body tissues and this portion is known as the bioavailable testosterone (BioT) (3).

Given the scenario described above, recommended practice would be to assess free testosterone by equilibrium dialysis and treat with testosterone if the free testosterone is low (4). The BioT by ammonium sulphate precipitation can also be used since this is a better marker of clinical status than TT alone. Epidemiological studies have confirmed that BioT correlates with parameters such as bone mineral density (5), sexual function (6), muscle mass (7) the development of generalised frailty in elderly African-Americans (8). The conventional testosterone assay measures all fractions of testosterone and does not make distinction between free testosterone, albumen-bound testosterone and SHBG-bound testosterone. A proportion of men including those with normal or borderline TT levels may have a low free testosterone or BioT if assays were readily available. In the absence of this measurement these men are unlikely to be offered androgen replacement.

The gold standard test of androgen status is an assay of free testosterone by equilibrium dialysis but determination of the bioavailable fraction of testosterone by ammonium sulphate precipitation is accurate, reliable and the techniques have a high degree of agreement (9). The bioavailable fraction of testosterone is calculated as a percentage of the TT, in this method testosterone is removed from the test serum by the addition of activated charcoal and replaced by $\left[{ }^{3} \mathrm{H}\right]$ testosterone, which binds to SHBG and other plasma proteins in equimolar concentrations as native testosterone. SHBG-bound testosterone is then precipitated out of the sample by the addition of cold ammonium sulphate. The remaining $\left[{ }^{3} \mathrm{H}\right]$ testosterone, comprising the unbound fraction and the fraction weakly bound to albumen are then measured using a beta counter. This value is converted to a percentage and comprises the bioavailable fraction of the TT (10). Equilibrium dialysis and BioT estimation are technically difficult techniques and are time consuming. The use of these techniques has been primarily in research laboratories.
The lack of readily available assays for free testosterone and BioT has forced clinicians to rely simply on the TT or to use calculated indices of biologically available such as the free androgen index, $\mathrm{FAI}=$ $\mathrm{TT} / \mathrm{SHBG} \times 100$ (11), or more complicated calculations of free testosterone such as those described by Nanjee and Wheeler (12) and Vermeulen (13). In addition, estimation of serum free testosterone by analogue assay is available. This technique should not be confused with BioT estimation by ammonium sulphate precipitation. The analogue assay performs relatively poorly when compared with equilibrium dialysis and is of doubtful value $(9,14)$.

In clinical practice the free androgen index (FAI) is probably the most widely used index of biological available testosterone, despite the fact that this index was modelled on a relatively small population of hirsute women $(n=30)$ and that prospective validation in men is limited. The other indices have also been modelled on relatively small populations of both sexes.

In this study we compared the relative merits of TT, SHBG, FAI and two other measures of free testosterone calculated from published formulae. Each assessment was evaluated for its ability to predict individual measures of BioT level in a large cohort of men. We have also derived and validated our own equation for calculating BioT from the TT and SHBG values. Finally, we have compared the efficacy of all these serum measures for diagnosing clinical hypogonadism.

\section{Material and methods}

The data were taken from a cross-sectional study of men undergoing elective coronary angiography at a tertiary cardiac referral centre between June 2000 and June 2002. Consecutive male patients were approached between 0800 and $0930 \mathrm{~h}$ and those consenting to enter the study completed a short questionnaire detailing their medical history and current medication. Height and weight were measured and body mass index (BMI) was calculated from weight $(\mathrm{kg}) /$ height $^{2}\left(\mathrm{~m}^{2}\right)$, and obesity was defined as a BMI $>30 \mathrm{~kg} / \mathrm{m}^{2}$. Ten millilitres of venous blood was taken and spun serum was frozen at $-20^{\circ} \mathrm{C}$ until assayed. Patients were excluded if they had a history of any form of malignancy, chronic inflammatory disease, myocardial infarction within the last 3 months, recent or current infection or uncontrolled heart failure, or if they were taking sex hormone manipulating therapy. Since inflammatory disorders have been shown to suppress serum testosterone levels, men were also excluded if they had a C-reactive protein level above $10 \mathrm{mg} / \mathrm{l}$. The local ethics committee approved the study.

Serum BioT was measured using an adaptation of the method described by Tremblay and Dube (10) and described in our previous work (15). Serum TT and 
SHBG were measured in duplicate by ELISA (DRG Instruments $\mathrm{GmbH}$, Marburg, Germany). The intraand inter-assay coefficients of variation of the assays were: $\mathrm{TT}<5 \%$ and $<6 \%$; SHBG $<9 \%$ and $<12 \%$ and BioT $<5 \%$ and $<8 \%$.

FAI was calculated by: TT/SHBG $\times 100$ (11). Percentage free testosterone (pmol/l) was calculated by the formula: $-2.38 \log _{10}(\mathrm{SHBG}+6.11)(12)$. Free testosterone (pmol/l) was calculated using a computer program to solve the third-order equation published and validated by Vermeulen et al. (13)

The normal range of testosterone varies between hospitals. At our institution, clear-cut androgen deficiency is diagnosed with strict criteria of a BioT level $\leq 2.5 \mathrm{nmol} / \mathrm{l}$ and $/$ or a $\mathrm{TT} \leq 7.5 \mathrm{nmol} / \mathrm{l}$. These strict arbitrary cut-off values are highly specific and this means that virtually all men are significantly hypogonadal by these criteria; by comparison other centres use a TT of $<12.0 \mathrm{nmol} / \mathrm{l}$ as a cut-off level, which is more sensitive but less specific. We confirmed the specificity of our criteria in clinical practice. All men who were classified hypogonadal by these criteria were invited back for assessment. By the end of February 2003, 97 accepted further endocrinological investigation and 92 completed an androgen deficiency questionnaire (16), which was positive in 86 (93\%). Of these 86 patients 81 were considered theoretically appropriate for androgen therapy by a consultant endocrinologist (T.H.J.). We believe these data confirm a clear relationship between our total/BioT values and symptoms of androgen deficiency since the positive predictive value of our values was $93 \%$ by symptoms and $88 \%$ by assessment from a consultant endocrinologist.

\section{Statistics and analysis}

Data were analysed using SPSS (version 11.5). Measured and calculated variables were correlated against BioT quoting Pearson's correlation coefficient. Individual and grouped variables were assessed as markers of BioT by single and multiple regression analysis, quoting the adjusted $R^{2}$. There is inherent variability of all hormone results proportional to their concentration and all data were therefore log transformed using a natural logarithm for all regression analyses. Transformation was confirmed by plotting a normal plot and checking standardised residuals. The diagnostic power of individual tests for predicting hypogonadism was displayed using receiver operator curves, using the presence of hypogonadism as a binary variable.

We performed three main analyses:

1. The ability of all currently available markers of androgen status to predict the level of BioT was compared in our cohort using regression analysis.

2. The data from our cohort was used to derive a formula that could be used to predict the level of BioT in a population. The cohort was therefore, divided randomly into a 'training set' (two-thirds of subjects), which was used to develop the model formula, and a 'validation set' (one-third of subjects) which was used to test it.

3. The ability of all the individual markers of androgen status (including our derived formula) to predict biochemical hypogonadism as defined at our centre was compared using receiver operator curves.

\section{Results}

Table 1 compares hormone parameters in their ability to determine the BioT level (analysis 1 ). The $P$ value indicates whether the relationship is significant and the $R$-value designates the closeness of fit of the relationship. This regression analysis demonstrates that the TT measure was the single best predictor of the BioT level in the whole population and in all subgroups. SHBG and FAI were consistently the worst predictors of BioT.

We used a regression model to develop an equation to calculate values of BioT; no physiological parameter significantly improved the regression model beyond the inclusion of TT and SHBG (analysis 2).

The equation was modelled on the training cohort $(n=715)$, using multiple linear regression analysis. $\beta$ coefficients were calculated from the regression model and applied to the corresponding values of $\mathrm{TT}$ and SHBG:

$\ln \mathrm{BioT}=-0.266+(0.955 \times \ln \mathrm{TT})-(0.228 \times \ln \mathrm{SHBG})$

( $\ln =$ natural $\log$, units are nmol/l). This equation was then tested on the 'validation set' $(n=357)$. The derived BioT values correlated significantly with the true values (Fig. 1). As a measure of agreement the difference between the derived and true values for BioT were plotted against zero using a Bland-Altman plot (Fig. 2) (17). This plot confirms a high level of agreement between true and derived values for BioT thus validating our equation. The equation was also prospectively tested on a separate cohort of 80 men with coronary disease who were investigated for androgen status; again, the true and derived values showed a high level of correlation and agreement (Fig. 3).

We then compared the values derived from our equation against the other measures and indices of androgen status (Table 2) using the validation cohort $(n=357)$. This analysis revealed that our equation was superior to the current methods of estimating biologically active testosterone.

Figure 4 show receiver operating characteristic (ROC) curves demonstrating the diagnostic performance of each assay in predicting biochemical hypogonadism. The TT was the best predictor of hypogonadism as defined at our centre in the whole population and the subgroup analyses. Table 3 shows the area under the 
Table 1 Summary of regression analyses indicating the relationship of different hormone parameters to the BioT level (nmol/l).

\begin{tabular}{|c|c|c|c|c|c|}
\hline Population & $n$ & Marker & $\boldsymbol{R}$ & $\boldsymbol{R}^{2}$ & $P$ \\
\hline \multirow[t]{5}{*}{ All men } & 1072 & TT* & 0.79 & 0.617 & $<0.001$ \\
\hline & & SHBG & 0.10 & 0.01 & $<0.001$ \\
\hline & & FAl & 0.40 & 0.159 & $<0.001$ \\
\hline & & $\%$ free testosterone & 0.76 & 0.58 & $<0.001$ \\
\hline & & Calculated free testosterone & 0.73 & 0.54 & $<0.001$ \\
\hline \multirow{5}{*}{ Obese men } & 282 & TT* & 0.81 & 0.65 & $<0.001$ \\
\hline & & SHBG & 0.06 & 0.003 & 0.33 \\
\hline & & $\mathrm{FAl}$ & 0.54 & 0.29 & $<0.001$ \\
\hline & & $\%$ free testosterone* & 0.81 & 0.65 & $<0.001$ \\
\hline & & Calculated free testosterone & 0.78 & 0.60 & $<0.001$ \\
\hline \multirow[t]{5}{*}{ Men with TT levels $<12 \mathrm{nmol} / \mathrm{l}$} & 471 & $\mathrm{TT}^{*}$ & 0.68 & 0.46 & $<0.001$ \\
\hline & & SHBG & 0.18 & 0.03 & $<0.001$ \\
\hline & & FAI & 0.48 & 0.23 & $<0.001$ \\
\hline & & $\%$ free testosterone & 0.66 & 0.44 & $<0.001$ \\
\hline & & Calculated free testosterone & 0.63 & 0.39 & $<0.001$ \\
\hline \multirow{5}{*}{ Men over 60 years } & 562 & TT* & 0.80 & 0.64 & $<0.001$ \\
\hline & & SHBG & 0.14 & 0.17 & $<0.01$ \\
\hline & & FAI & 0.52 & 0.27 & $<0.001$ \\
\hline & & $\%$ free testosterone & 0.75 & 0.56 & $<0.001$ \\
\hline & & Calculated free testosterone & 0.72 & 0.52 & $<0.001$ \\
\hline \multirow{5}{*}{ Smokers } & 150 & $\mathrm{TT} *$ & 0.74 & 0.54 & $<0.001$ \\
\hline & & SHBG & 0.17 & 0.02 & 0.03 \\
\hline & & FAl & 0.37 & 0.13 & $<0.001$ \\
\hline & & $\%$ free testosterone & 0.69 & 0.47 & $<0.001$ \\
\hline & & Calculated free testosterone & 0.64 & 0.41 & $<0.001$ \\
\hline \multirow[t]{5}{*}{ Hypertensive men } & 482 & $\mathrm{TT}^{*}$ & 0.78 & 0.61 & $<0.001$ \\
\hline & & SHBG & 0.09 & 0.01 & $<0.6$ \\
\hline & & FAl & 0.51 & 0.26 & $<0.001$ \\
\hline & & $\%$ free testosterone & 0.77 & 0.60 & $<0.001$ \\
\hline & & Calculated free testosterone & 0.75 & 0.56 & $<0.001$ \\
\hline \multirow{5}{*}{ Statin takers } & 714 & $\mathrm{TT} *$ & 0.76 & 0.58 & $<0.001$ \\
\hline & & SHBG & 0.03 & 0.001 & 0.50 \\
\hline & & $\mathrm{FAl}$ & 0.52 & 0.27 & $<0.001$ \\
\hline & & $\%$ free testosterone ${ }^{*}$ & 0.76 & 0.58 & $<0.001$ \\
\hline & & Calculated free testosterone & 0.73 & 0.54 & $<0.001$ \\
\hline \multirow[t]{5}{*}{ Diabetic men } & 132 & $\mathrm{TT}^{\star}$ & 0.79 & 0.62 & $<0.001$ \\
\hline & & SHBG & 0.04 & 0.002 & 0.6 \\
\hline & & $\mathrm{FAl}$ & 0.50 & 0.24 & $<0.001$ \\
\hline & & $\%$ free testosterone & 0.78 & 0.61 & $<0.001$ \\
\hline & & Calculated free testosterone & 0.74 & 0.54 & $<0.001$ \\
\hline
\end{tabular}

* Best test for each population.

The $P$ value determines whether the relationship is significant and the $R^{2}$ value designates the closeness of fit of the relationship.

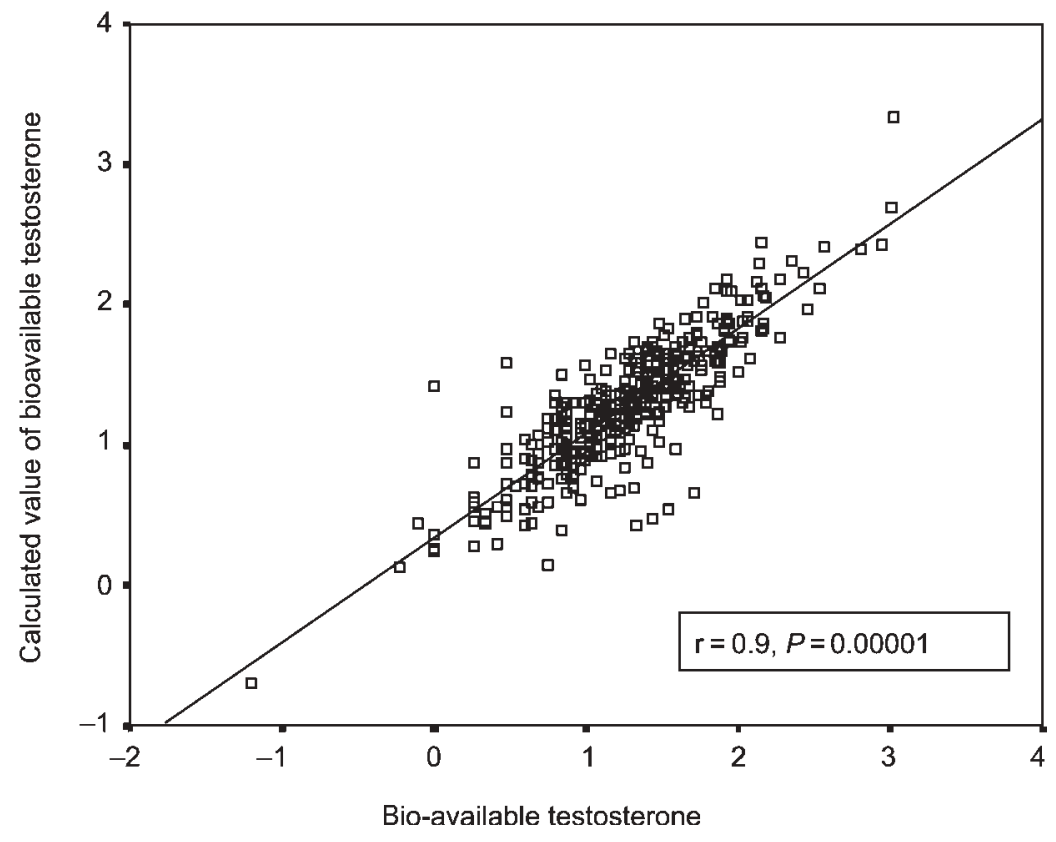

Figure 1 The correlation between actual BioT $(\mathrm{nmol} / \mathrm{l})$ and BioT calculated from the regression equation: $\ln B T=-0.266+(0.955 \times$ $\ln T \mathrm{~T})-(0.228 \times \operatorname{InSHBG})$ in the validation set of patients $(n=357)$. 

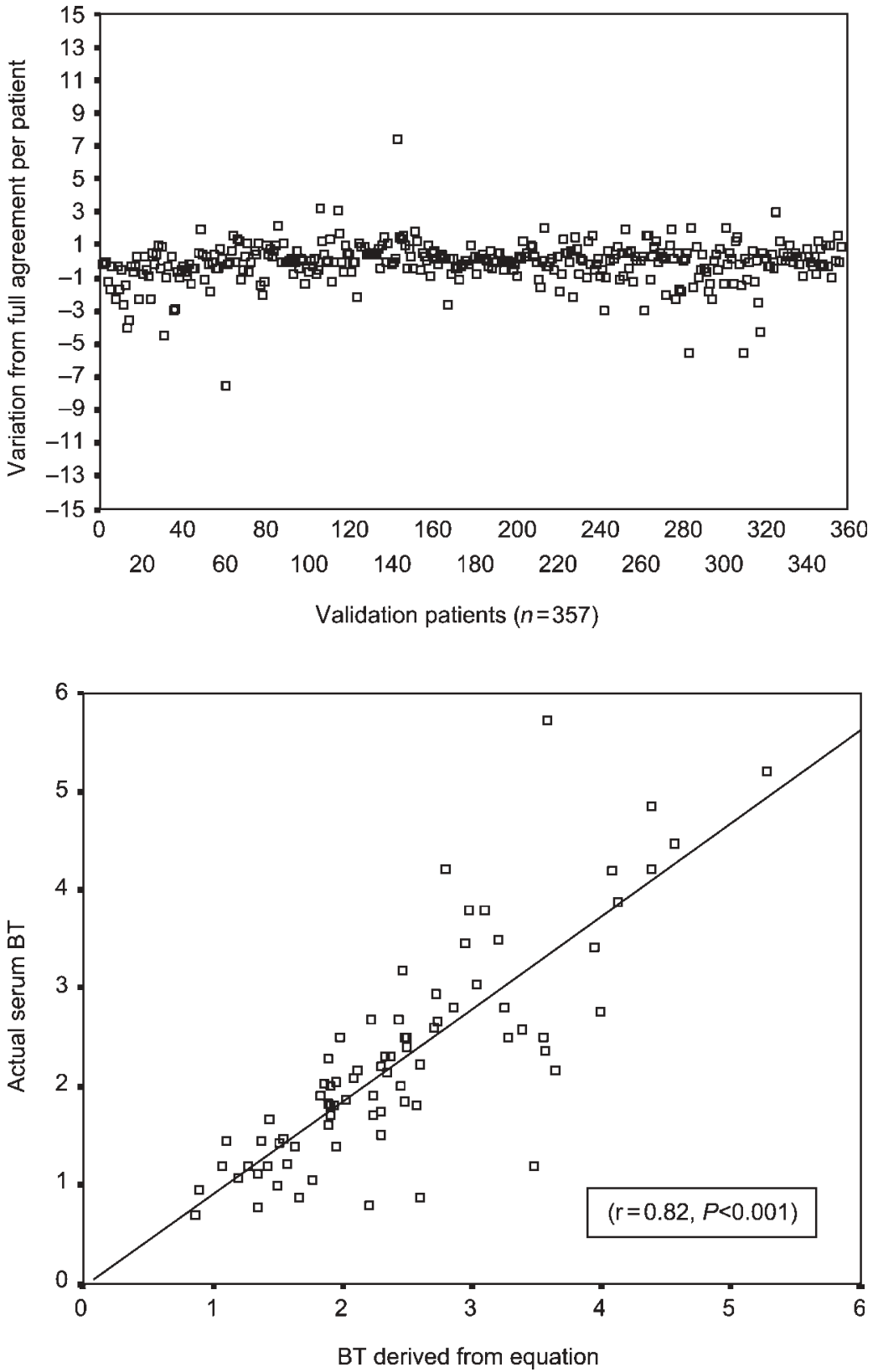

Figure 2 A Bland-Altman graph showing the level of agreement between serum BioT $(\mathrm{nmol} / \mathrm{l})$ and BioT calculated from the regression equation: $\ln B T=-0.266+(0.955 \times$ $\operatorname{lnTT})-(0.228 \times \ln S H B G)$ in the validation cohort $(n=357)$. Absolute agreement would have a variation of zero.
Figure 3 The correlation between serum BioT (nmol/l) and BioT calculated from the regression equation: InBioT $=-0.266+$ $(0.955 \times \ln T \mathrm{~T})-(0.228 \times \operatorname{lnSHBG})$ in 80 men with coronary disease.
Table 2 A regression analysis comparing our calculated values for BioT (BTcalculated) from the regression equation against other measures and indices on the validation group $(n=357)$.

\begin{tabular}{lcc}
\hline Measure & $\boldsymbol{R}^{2}$ & $\boldsymbol{P}$ \\
\hline TT & 0.68 & $<0.0001$ \\
BTcalculated & 0.73 & $<0.0001$ \\
FAl & 0.26 & $<0.0001$ \\
Free testosterone & 0.55 & $<0.0001$ \\
$\%$ free testosterone & 0.59 & $<0.0001$
\end{tabular}

The $P$ value determined whether the relationship is significant. The $R^{2}$ value determines the closeness of fit of this relationship. curve for each the ROC curves. These values are an indicator of how accurate a particular measure is at predicting hypogonadism since sensitivity and the specificity are included in the analysis. A test with a score of 1 indicates $100 \%$ sensitivity and specificity, whereas a test with a score of 0.5 provides no useful diagnostic information. The levels of BioT derived from our equation were compared with the other tests of androgen status. Of the current available measures the TT was consistently the superior test as an indicator of hypogonadism. However, the derived values for BioT from our equation demonstrated slightly better predictive powers. FAI and SHBG were consistently the worst predictors of hypogonadism. 
(a)

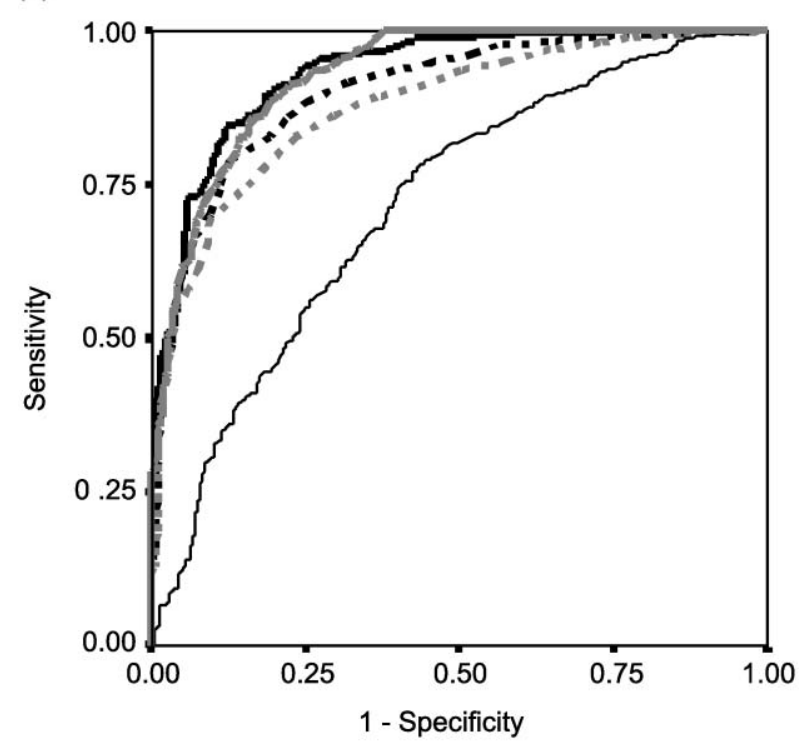

(c)

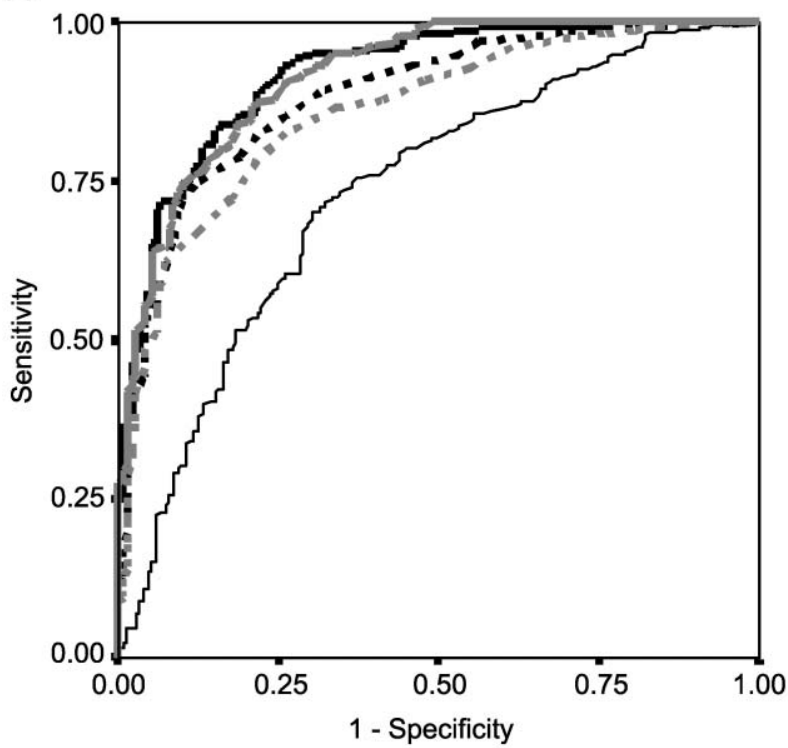

(b)

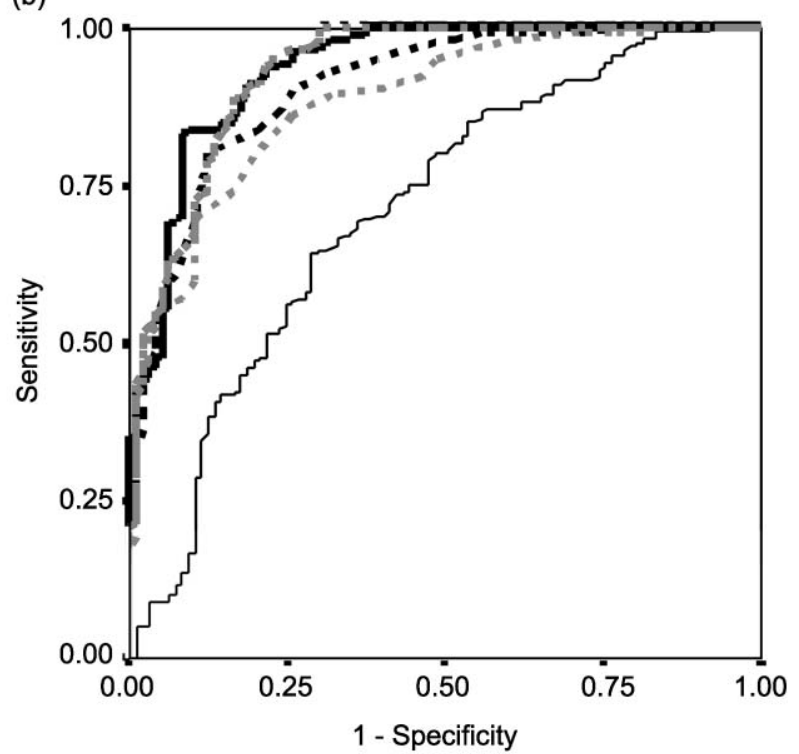

(d)

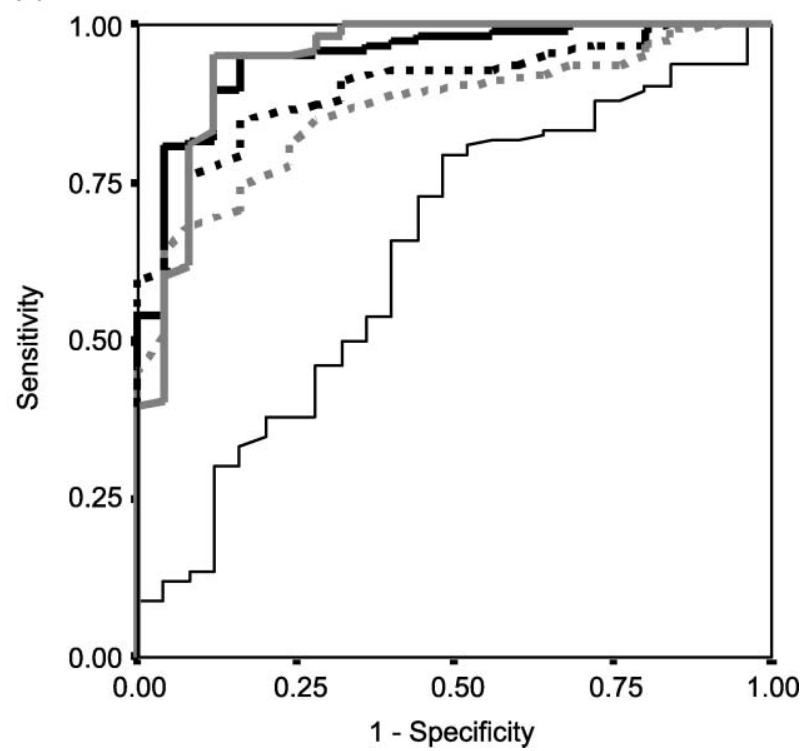

Figure 4 Receiver operating characteristic (ROC) curves demonstrating the diagnostic performance of each assay in predicting biochemical hypogonadism. Btcalculated, BioT calculated by the derived by the regression equation; FTnw, free testosterone as calculated by the method of Nanjeee and Wheeler; FTv, free testosterone as calculated by the method of Vermeulen, FAl = free androgen index, TT $=$ total testosterone. (a) Whole population, $n=1072$, (b) obese men, $n=282$ (c) men age $>60$ years, $n=562$, (d) smokers, $n=150$, (e) diabetes, $n=132$, (f) serum $7.5<\mathrm{TT}<12 \mathrm{nmol} / \mathrm{l}, n=317$.

Men with TT levels above $7.5 \mathrm{nmol} / \mathrm{l}$ but below $12 \mathrm{nmol} / \mathrm{l}$ were also assessed as a subgroup, since this group provides an area of genuine clinical doubt. A BioT assay would be particularly useful in this group, but is usually not available. We therefore used our derived values of BioT and compared these with the other measurements. Overall in this group $(n=317)$ the calculated indices of androgen status including our derived measurements were of equivalent efficacy.

\section{Conclusions}

In this paper we have addressed three main issues. Firstly, what is currently the best marker of BioT available to clinicians? Our data show clearly that TT is consistently the best predictor the BioT level in all populations and sub-groups. Secondly and perhaps not surprisingly the TT is also the best predictor of biochemical hypogonadism. Thirdly when there is genuine clinical doubt, for example when TT is between 7.5 and 
(e)

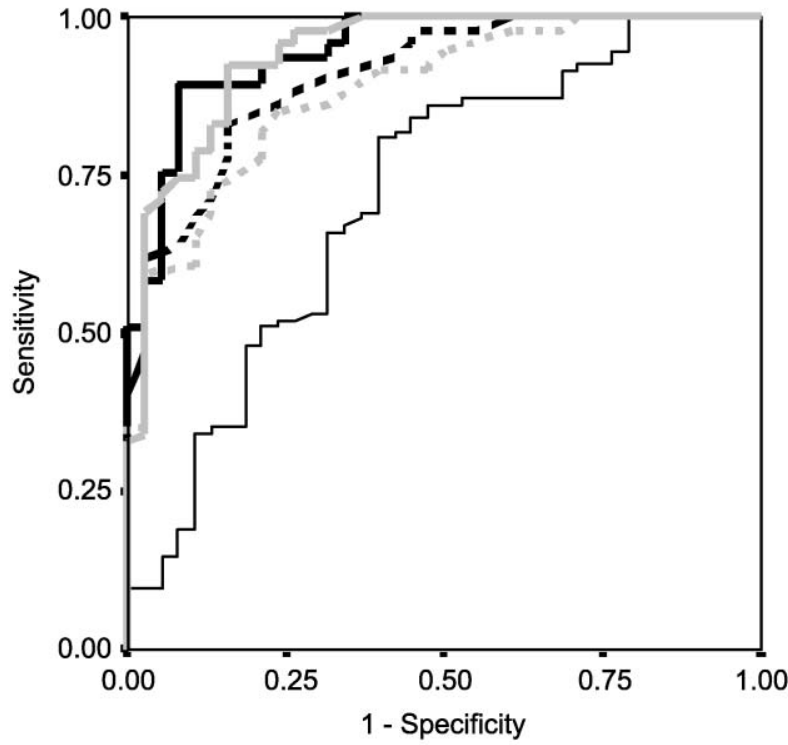

(f)

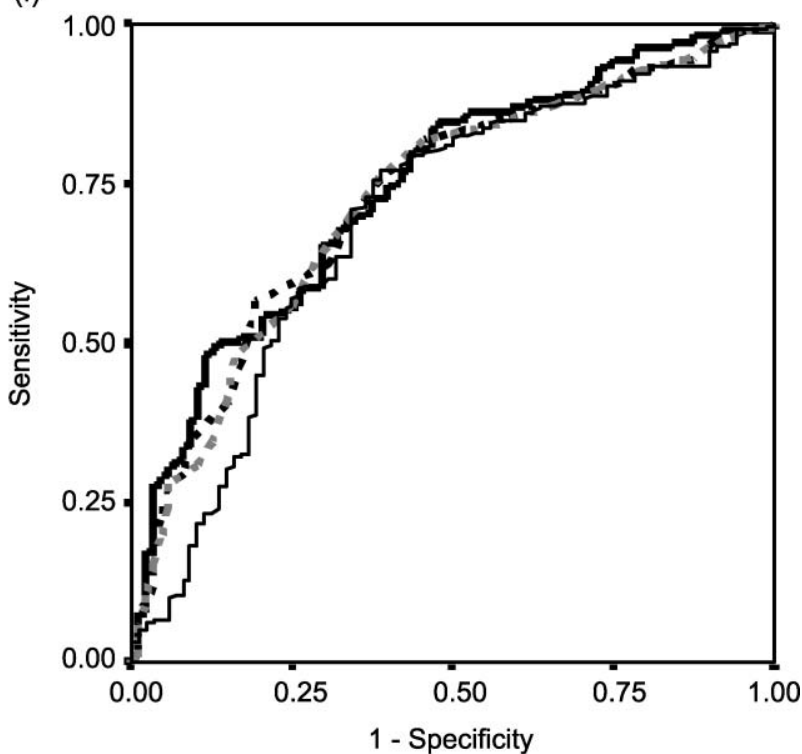

Source of the Curve

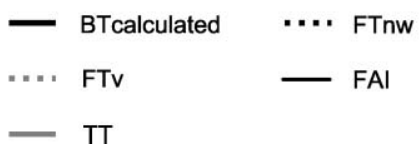

Figure 4 Continued.

Table 3 Summary of the area under the receiver operating curves of each serum assessment or index. The closer the area to 1 $(100 \%)$ the greater the power to diagnose hypogonadism.

\begin{tabular}{|c|c|c|c|}
\hline Population & $n$ & Marker & Area under the curve \\
\hline \multirow[t]{5}{*}{ All men } & 1072 & Total testosterone & 0.93 \\
\hline & & $\mathrm{FAl}$ & 0.72 \\
\hline & & $\%$ free testosterone & 0.91 \\
\hline & & Calculated free testosterone & 0.88 \\
\hline & & BTcalculated & 0.94 \\
\hline \multirow[t]{4}{*}{ Obese men } & 282 & Total testosterone & 0.93 \\
\hline & & $\mathrm{FAl}$ & 0.71 \\
\hline & & $\%$ free testosterone & 0.91 \\
\hline & & Calculated free testosterone & 0.89 \\
\hline \multirow[t]{4}{*}{ Men over 60 years } & 562 & Total testosterone & 0.92 \\
\hline & & $\mathrm{FAl}$ & 0.73 \\
\hline & & $\%$ free testosterone & 0.89 \\
\hline & & Calculated free testosterone & 0.86 \\
\hline \multirow[t]{4}{*}{ Smokers } & 150 & Total testosterone & 0.94 \\
\hline & & $\mathrm{FAl}$ & 0.64 \\
\hline & & $\%$ free testosterone & 0.90 \\
\hline & & Calculated free testosterone & 0.87 \\
\hline \multirow[t]{4}{*}{ Diabetic men } & 132 & Total testosterone & 0.94 \\
\hline & & FAI & 0.72 \\
\hline & & $\%$ free testosterone & 0.91 \\
\hline & & Calculated free testosterone & 0.88 \\
\hline \multirow[t]{5}{*}{ Men with $\mathrm{TT}<12 \mathrm{nmol} / \mathrm{l}$ and $>7.5 \mathrm{nmol} / \mathrm{l}$} & 317 & Total testosterone & 0.63 \\
\hline & & FAI & 0.74 \\
\hline & & $\%$ free testosterone & 0.75 \\
\hline & & Calculated free testosterone & 0.75 \\
\hline & & BTcalculated & 0.75 \\
\hline
\end{tabular}


$12 \mathrm{nmol} / \mathrm{l}$, calculated indices of free testosterone or BioT are helpful and improve the diagnostic yield but each appear to be of equivalent efficacy.

We used two-thirds of our data to develop a novel formula to calculate the level of BioT given the standard laboratory measures of TT and SHBG. Data derived with this equation were validated on the remaining third. We went on to compare our derived values with the other measures previously tested. In this analysis our novel formula performed marginally better overall.

\section{Discussion}

There are no clear and simple measures of androgen status, especially in patients where conventional testosterone assays yield borderline results. In this scenario more sensitive and specific tests of androgen status are recommended (4), the gold standard measure of androgen status is the equilibrium dialysis estimation of free testosterone but the bioavailable fraction determination of TT provides equally accurate values. The two techniques have a high degree of concordance (9), however these assays currently remain research tools and are not widely available. In this paper, for the first time, we have compared conventional serum and calculated measures of androgen status with BioT and found that in general TT remains the single best marker of androgen status. However using an equation derived from regression analysis of our data we were able to slightly improve on current calculated indices. Calculated methods of free testosterone or BioT do not offer greater sensitivity or specificity in predicting hypogonadism compared with a simple measure of TT unless the TT value is borderline. In this event any of the current methods of estimating free testosterone or BioT have similar efficacy. FAI was consistently the worst predictor of hypogonadism and BioT, this index appears to have a limited role in this cohort. These data are potentially useful to practising physicians, there does not appear to be any advantage in offering tests of androgen status beyond a simple TT. In borderline cases an assessment of free testosterone by equilibrium dialysis is recommended or BioT by ammonium sulphate is as useful; however our data suggest that any of the calculated indices of free testosterone or our index of BioT would be as useful.

\section{Limitations}

We did not measure serum albumen concentration in this cohort of men. The bioavailable assay determines albumen-bound testosterone but not the concentration of albumen. Calculated indices of free testosterone are available using serum albumen as a coefficient, consequently we were unable to test the accuracy of these indices in this paper.
The men in the study cohort were not randomly selected; they were all men with either manifest or suspected cardiac disease recruited prior to diagnostic coronary angiography. The prevalence of hypogonadism by our strict criteria in this group was $23.4 \%$, a figure at least three times the background community prevalence. It could be argued that this reduces the power of our calculations and that our results would only apply to a similar cohort. However we believe that the sample selected actually strengthens our results since the sample was taken from the lower tail of the population as determined by serum testosterone. This is the group of patients in which androgen deficiency is more prevalent and diagnosis of androgen deficiency more difficult due to borderline TT values. Therefore our results are more applicable precisely to the population of interest - subjects with low or borderline low serum testosterone, an unforeseen advantage.

\section{References}

1 Rhoden EL \& Morgentaler A. Risks of testosterone-replacement therapy and recommendations for monitoring. New England Journal of Medicine $20043 \mathbf{3 0 5} 482-492$.

2 Morales A \& Lunenfeld B. Investigation, treatment and monitoring of late-onset hypogonadism in males. Official recommendations of ISSAM. International Society for the Study of the Aging Male. Aging Male 200252 74-86.

3 Channer KS \& Jones TH. Cardiovascular effects of testosterone: implications of the 'male menopause'? Heart 200389 121-122.

4 Snyder PJ. Hypogonadism in elderly men - what to do until the evidence comes. New England Journal of Medicine 2004350 440-442.

5 van den Beld AW, de Jong FH, Grobbee DE, Pols HA \& Lamberts SW. Measures of bioavailable serum testosterone and estradiol and their relationships with muscle strength, bone density, and body composition in elderly men. Journal of Clinical Endocrinology and Metabolism $2000 \mathbf{8 5} 3276-3282$.

6 Nilsson PM, Moller L \& Solstad K. Adverse effects of psychosocial stress on gonadal function and insulin levels in middle-aged males. Journal of Internal Medicine 1995237 479-486.

7 Iannuzzi-Sucich M, Prestwood KM \& Kenny AM. Prevalence of sarcopenia and predictors of skeletal muscle mass in healthy, older men and women. Journals of Gerontology. Series A, Biological Sciences and Medical Sciences 200257 M772-M777.

8 Perry HM 3rd, Miller DK, Patrick P \& Morley JE. Testosterone and leptin in older African-American men: relationship to age, strength, function, and season. Metabolism $2000 \mathbf{4 9}$ 1085-1091.

9 Vermeulen A, Verdonck L \& Kaufman JM. A critical evaluation of simple methods for the estimation of free testosterone in serum. Journal of Clinical Endocrinology and Metabolism $1999 \mathbf{8 4}$ 3666-3672.

10 Tremblay RR \& Dube JY. Plasma concentrations of free and nonTeBG bound testosterone in women on oral contraceptives. Contraception 197410 599-605.

11 Wilke TJ \& Utley DJ. Total testosterone, free-androgen index, calculated free testosterone, and free testosterone by analog RIA compared in hirsute women and in otherwise-normal women with altered binding of sex-hormone-binding globulin. Clinical Chemistry $1987331372-1375$.

12 Nanjee MN \& Wheeler MJ. Plasma free testosterone - is an index sufficient? Annals of Clinical Biochemistry 198522 387-390. 
13 Vermeulen A. Testosterone in plasma. A physiopathological study. Verhandelingen - Koninklijke Academie voor Geneeskunde van Belgie 197335 95-180.

14 Winters SJ, Kelley DE \& Goodpaster B. The analog free testosterone assay: are the results in men clinically useful? Clinical Chemistry $1998 \mathbf{4 4} 2178-2182$.

15 English KM, Mandour O, Steeds RP, Diver MJ, Jones TH \& Channer KS. Men with coronary artery disease have lower levels of androgens than men with normal coronary angiograms. European Heart Journal 200021 890-894.
16 Morley JE, Charlton E, Patrick P, Kaiser FE, Cadeau P, McCready D et al. Validation of a screening questionnaire for androgen deficiency in aging males. Metabolism 200049 1239-1242.

17 Bland JM \& Altman DG. Measurement error. BMJ 1996313744.

Received 30 January 2004

Accepted 21 April 2004 